\title{
Detection and Measurement of Intracranial Aneurysm Compared between Magnetic Resonance Intracranial Black Blood Vessel Imaging and Gold Standard Cerebral Digital Subtraction Angiography
}

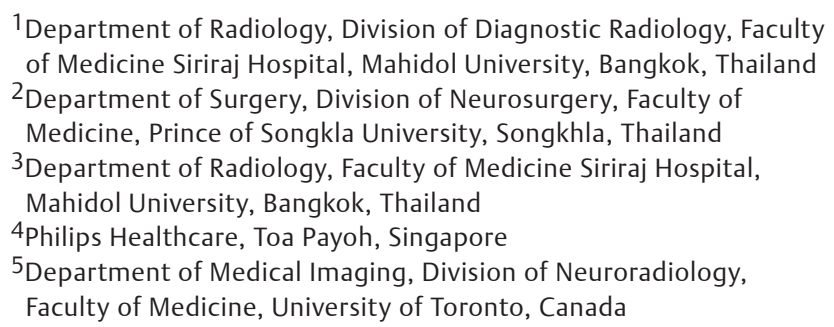

Dittapong Songsaeng ${ }^{1}$ Ittichai Sakarunchai ${ }^{2} \quad$ Sakun Mongkolnaowarat ${ }^{1}$ Sasithorn Harmontree Prapaporn Pornpunyawut ${ }^{3}$ Anek Suwanbundit ${ }^{3}$ Shuo Zhang ${ }^{4}$ Timo Krings ${ }^{5}$

\begin{abstract}
Address for correspondence Dittapong Songsaeng, MD, Department of Radiology, Division of Diagnostic Radiology, Faculty of Medicine Siriraj Hospital, Mahidol University, 2 Wang lang Road, Bangkok Noi, Bangkok 10700, Thailand (e-mail: songsaengmek@gmail.com).
\end{abstract}

Abstract


Keywords
- intracranial aneurysm
- black blood vessel
imaging
- cerebral digital
subtraction
angiography
- measurement
- 3 D TOF MRA

Background Magnetic resonance intracranial black blood vessel imaging (MR-IBBVI) is a new noninvasive method for evaluating intracranial vessel wall pathology. No previous studies have investigated the efficacy of MR-IBBVI to determine aneurysm size. We aimed to identify the precise diagnosis of MR-IBBVI for the detection and measurement of intracranial aneurysm compared with gold standard cerebral digital subtraction angiography (cDSA).

Materials and Methods The retrospective study collected patients of precoiled or postcoiled intracranial aneurysm who were treated at our institute from January 2012 to June 2019 and who had MR-IBBVI, CDSA imaging, and/or three-dimensional timeof-flight sequence of magnetic resonance angiography. The sensitivity and specificity of aneurysm detection by MR-IBBVI and the accuracy of MR-IBBVI for measuring the aneurysm and vessel size were calculated.

Results One hundred and twenty patients (61\% female) with 132 aneurysms were included into this study. The mean aneurysm size was $5.3 \mathrm{~mm}$ (range: 2.2-22.6). Sensitivity and specificity of MR-IBBVI to detect a small aneurysm were 98.74 and 91.21\%, respectively. No statistically significant results were observed between MR-IBBVI and DSA for aneurysm detection or any of the evaluated measurement parameters.

Conclusion MR-IBBVI is an accurate and highly sensitive method to detect and evaluate the size of an intracranial aneurysm both before and after coiling.

\section{Introduction}

The prevalence of intracranial aneurysms is $\sim 4 \%$ in the general population. ${ }^{1}$ Most of the aneurysms occur in people aged 40 to 60 years. ${ }^{2}$ Subarachnoid hemorrhage is high incidence of mortality rate ranging from 25 to $50 \%{ }^{3}$ Cerebral digital

DOI https://doi.org/ 10.1055/s-0040-1714042 ISSN 0976-3147. subtraction angiography (cDSA) is the gold standard for intracranial aneurysm diagnosis and follow-up; however, the risks associated with CDSA are higher than other imaging modalities. Nowadays, there is some interest in magnetic resonance intracranial black blood vessel imaging (MR-IBBVI) using for the assessment of aneurysm wall inflammation and wall 
thickness that may help predict the risk of aneurysm rupture. ${ }^{4}$ No previous studies have investigated the efficacy of MR-IBBVI to determine the size of intracranial aneurysm. Accordingly, the aim of this study was to determine the precise diagnosis of MR-IBBVI for the detection and measurement of intracranial aneurysm compared with gold standard cDSA.

\section{Materials and Methods}

\section{Patients}

This retrospective study included patients with precoiled or postcoiled intracranial aneurysm who were treated at our institute from January 2012 to June 2019. All patients included in the study had undergone MR-IBBVI, cDSA imaging, and/or three-dimensional time-of-flight sequence of magnetic resonance angiography (3D TOF MRA). Demographic data, stroke symptoms, diagnosis, gender, age, lesion location, and shape of aneurysm were collected from the patient medical records and our center's picture archiving and communication system. This study protocol was approved by the institutional review board.

\section{Imaging}

\section{Magnetic Resonance Intracranial Black Blood Vessel Imaging}

MR-IBBVI was performed using Philips Ingenia 3.0T software 5.1.7.2 and software 5.3.1.0 on 3D proton-density imaging, T1-weighted (T1W), T2-weighted (T2W), contrast enhanced T1W-VISTA/Gd using these parameters: TR 700 milliseconds; TE 33 milliseconds; flip angle 90; matrix 268x336; field of view 280x251 mm; section thickness $0.75 \mathrm{~mm}$; and number of signal averages (NSA) 2.

\section{Cerebral Digital Subtraction Angiography}

Biplane cDSA system (GE, IGS630; workstation, AW version 4.6) was performed using these parameters: matrix 2D 1024x1024 and matrix 3D 512x512. Transfemoral approach was performed for selective vessels study. Imaging was performed in the anteroposterior and Caldwell views. The largest diameter of the aneurysms was recorded on the workstation from the 3D images.

\section{Three-Dimensional Time-of-Flight Sequence of Magnetic} Resonance Angiography

MR imaging was performed by Philips Ingenia 3.0T software 5.1.7.2 and software 5.3.1.0 scanner with an axis gradient system. The standard image protocol was designed for $\mathrm{T} 2 \mathrm{~W}$ spin-echo sequence and fluid-attenuated sequence (TR 25 milliseconds; TE 3.5 milliseconds; flip angle 20; matrix 500x332; section thickness $1.2 \mathrm{~mm}$; NSA 1). No contrast material was used in the 3D TOF MRA.

\section{Image Interpretation}

The size of the aneurysm was ascertained by measuring the maximum width and length in millimeters in the same plane for each of the three evaluated imaging modalities (cDSA, MR-IBBVI, and 3D TOF MRA). Intraluminal diameter of normal parent artery was measured for technical reference in the same plane and location of the available internal carotid artery (ICA), anterior cerebral artery (ACA), middle cerebral

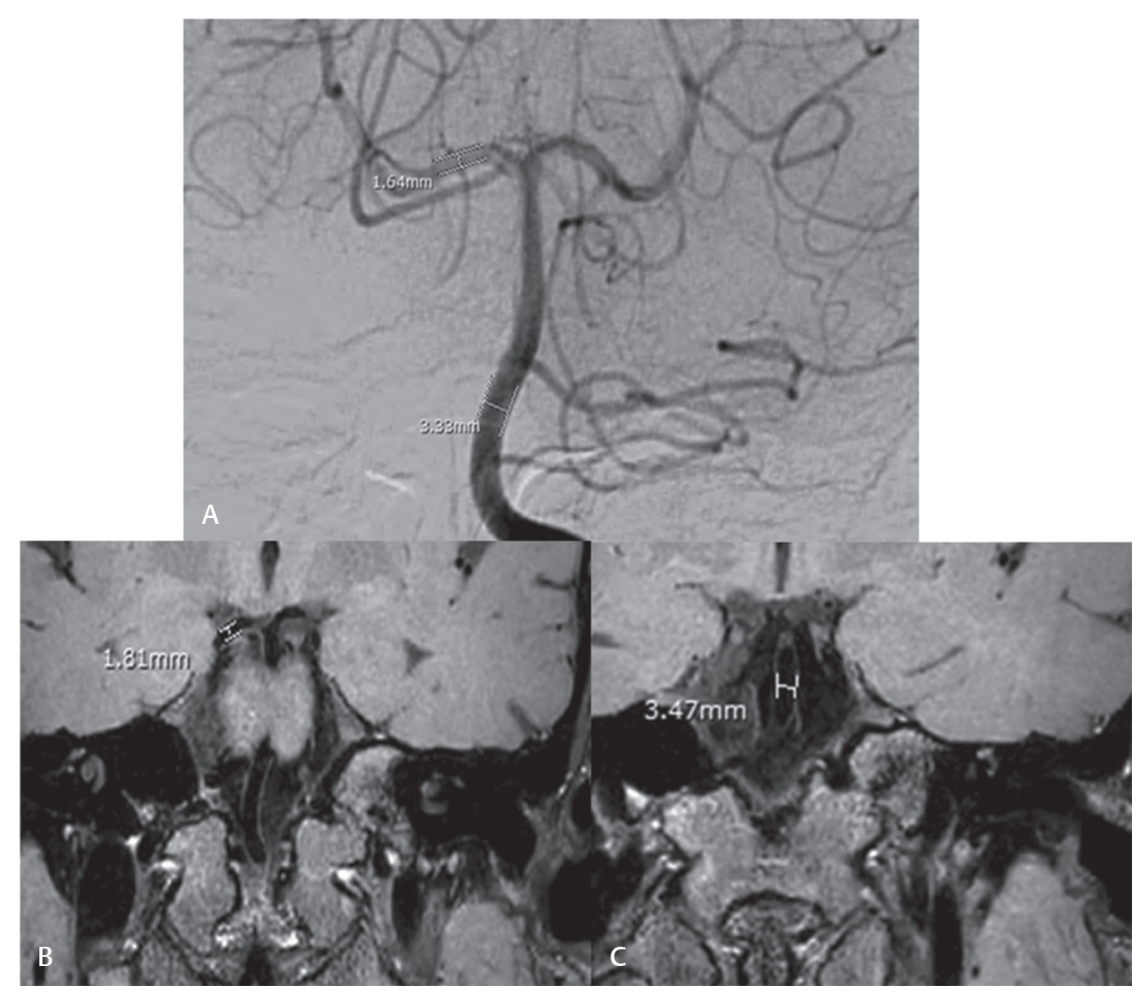

Fig. 1 Technical reference of the normal diameter of different vessels in the same plane and location of the internal carotid artery, anterior cerebral artery, middle cerebral artery, posterior cerebral artery, basilar artery, and/or vertebral artery. (A) Digital subtraction angiography. (B and C) Magnetic resonance black blood vessel imaging. 
artery (MCA), posterior cerebral artery (PCA), basilar artery (BA), and/or vertebral artery (VA) ( - Fig. 1). The shape of each aneurysm was categorized for three groups: residual of aneurysmal neck, fusiform-shape, and saccular-shaped aneurysm.

\section{Statistical Analysis}

Descriptive data was used to analyze demographic characteristics, such as age, gender, symptom, pretreatment, and posttreatment. Quantitative data are presented as mean \pm standard deviation (SD) or median (min, max). Qualitative data are given as frequency and percentage. The statistical analysis of the aneurysm size was performed using Student's $t$-test. The reference for significant aneurysm size was performed by a pilot study using the SD of difference $=1.4, \alpha$ $=0.01$, and $90 \%$ power using PASS software version 14.0 .7 (NCSS, LLC, Kaysville, Utah, United States). The results of that analysis revealed no significant difference in the measurement of aneurysm size within $1 \mathrm{~mm}$ by 3D cDSA versus MR-IBBVI. The statistical analysis was done by Pearson's chi-squared and Fisher's exact test for qualitative variables. A two-tailed $p<0.05$ was considered statistically significant. Statistical analysis was performed using SPSS Statistics version 18 (SPSS, Inc., Chicago, Illinois, United States).

\section{Results}

MR-IBBVI and CDSA were performed from January 2012 to June 2019 in 121 patients. One patient was excluded because one aneurysm had motion artifact. Data from the remaining 120 patients with 132 aneurysms were entered into the study. There were 47 males (39.1\%) and 73 females (60.8\%) and the age ranged from 34 to 77 years (average 58 years). The frequencies of intracranial aneurysm locations showed $20.8 \%$ at left ICA, $15 \%$ at right ICA and BA, 2.5\% at left VA, $4.3 \%$ at right VA, $5.8 \%$ at ACA, $24.9 \%$ at both MCAs, and $12.5 \%$ at both PCAs. The shapes of the aneurysms were classified as residual neck aneurysm (8.3\%), fusiform-shaped aneurysm (11.7\%), and saccular-shaped aneurysm (80.0\%) (-Table 1).

\section{Aneurysm Size}

The average size of the aneurysms was $5.04 \mathrm{~mm}$ in width (range: $0.81-22.38 \mathrm{~mm}$ ) and 8.53 in length (range: $1.5-43.41 \mathrm{~mm}$.) of cDSA. There were no statistically significant differences in either the mean widths or mean lengths of the intracranial vessels on MR-IBBVI compared with CDSA and 3D TOF MRA with $95 \%$ confidence interval (CI) (-Table 2). The accuracy of MR-IBBVI showed $80.6 \%$ and no statistically significant differences in measurement between MR-IBBVI and CDSA ( $\mathrm{SD}=1.4, \alpha=0.01$, power $90 \%$ ). However, the accuracy of 3D TOF MRA showed $73.3 \%$ in width and $70 \%$ in length and no statistically significant differences in measurement between 3D TOF MRA and CDSA ( - Table 3 ). Of all aneurysms on 3D TOF MRA, the size of $15 \%$ of the aneurysms could not truly be evaluated due to the inability to separate thrombosis associated with either coil mesh or regrowth aneurysm. Using a $1 \mathrm{~mm}$ difference in size criteria, no significant differences in width and length of the aneurysms were measured on
Table 1 Demographic data analysis including sex, treatment, shape, location, and size

\begin{tabular}{|l|l|l|}
\hline & Frequency & Percentage \\
\hline Sex & 120 & 100 \\
\hline Male & 47 & 39.1 \\
\hline Female & 73 & 60.8 \\
\hline Imaging follow-up & & \\
\hline Pre-treatment & 132 & 100 \\
\hline Posttreatment & 120 & 83.3 \\
\hline Aneurysm morphology & & \\
\hline Residual neck & 10 & 8.3 \\
\hline Fusiform & 14 & 11.7 \\
\hline Saccular & 96 & 80 \\
\hline Location & & \\
\hline Rt. ICA & 18 & 15 \\
\hline Lt. ICA & 25 & 20.8 \\
\hline ACA & 7 & 5.8 \\
\hline Rt. MCA & 13 & 10.8 \\
\hline Lt. MCA & 16 & 13.3 \\
\hline Rt. PCA & 8 & 6.7 \\
\hline Lt. PCA & 7 & 5.8 \\
\hline BA & 18 & 15 \\
\hline Rt. VA & 5 & 4.3 \\
\hline Lt. VA & 3 & 2.5 \\
\hline 5. Size & & \\
\hline <3 mm & 17 & 14 \\
\hline 3-7 mm & 50 & 42 \\
\hline >7 mm & 53 & 44 \\
\hline Ab & & \\
\hline
\end{tabular}

Abbreviations: ACA, anterior cerebral artery; BA, basilar artery; ICA, internal carotid artery; Lt., left; MCA, middle cerebral artery; mm, millimeter; PCA, posterior cerebral artery; Rt., right; VA, vertebral artery.

MR-IBBVI versus CDSA (mean difference $0.60 \mathrm{~mm}$., SD: 0.42, $95 \% \mathrm{CI} 0.37,0.65$ in width and mean difference 0.47 , SD: 0.62 , 95\% CI: 0.42, 0.93 in length). There was no statistically significant differences between 3D TOF MRA versus 3D cDSA but a relatively higher error in 3D TOF MRA compared with MR-IBBVI reported a mean difference of $0.75 \mathrm{~mm}$ (SD: 0.67, $95 \%$ CI: $0.47,1.21)$ in width and a mean difference of 0.69 (SD: $0.46,95 \%$ CI: 0.56, 1.13) in length (- Table 3 ). The number of disagreements between MR-IBBVI and CDSA seemingly occurred with residual neck aneurysms $40 \%$ (4/10 cases) regardless of the size of the aneurysms, but the results were not statistically significant.

\section{Vessel Size}

The accuracy for measurement of vessel size on MR-IBBVI was $100 \%$. No statistically significant differences between MR-IBBVI versus CDSA were observed (Student's $t$-test, 95\% CI minimum difference $0.03 \mathrm{~mm}$, maximum difference $0.76 \mathrm{~mm})$. 
Table 2 Aneurysm size measured by cDSA, MR-IBBVI, and 3D TOF MRA

\begin{tabular}{|l|l|l|l|l|l|l|}
\hline \multirow{2}{*}{} & \multicolumn{2}{|c|}{ CDSA } & \multicolumn{2}{c|}{ MR-IBBVI } & \multicolumn{2}{c|}{ 3D TOF MRA } \\
\cline { 2 - 8 } & Width & Length & Width & Length & Width & Length \\
\hline Mean (mm) & 5.04 & 8.53 & 5.29 & 8.67 & 5.31 & 8.72 \\
\hline Standard error 95\% Cl (mean) & 0.75 & 1.23 & 0.75 & 1.23 & 0.80 & 1.34 \\
\hline Lower bound & 3.34 & 6.26 & 3.76 & 5.78 & 3.38 & 5.62 \\
\hline Upper bound & 6.72 & 11.38 & 7.3 & 10.98 & 7.2 & 13.14 \\
\hline Median (mm) & 3.6 & 5.15 & 3.08 & 4.36 & 4.55 & 5.51 \\
\hline SD & 4.26 & 8.47 & 4.54 & 8.64 & 4.39 & 8.68 \\
\hline Minimum & 0.81 & 1.5 & 1.1 & 1.34 & 1.27 & 1.82 \\
\hline Maximum & 22.38 & 43.41 & 22.97 & 41.65 & 16.98 & 42.08 \\
\hline Range & 23.22 & 40.37 & 20.13 & 37.23 & 6.54 & 39.28 \\
\hline
\end{tabular}

Abbreviations: 3D TOF MRA, 3-dimensional time-of-flight magnetic resonance angiography; cDSA, cerebral digital subtraction angiography; mm, millimeter; MR-IBBVI, magnetic resonance intracranial black blood vessel imaging; SD, standard deviation.

Table 3 Comparative differences between MR-IBBVI and cDSA

\begin{tabular}{|l|l|l|l|l|}
\hline \multirow{2}{*}{} & \multicolumn{2}{|c|}{ MR-IBBVI } & \multicolumn{2}{c|}{ 3D TOF MRA } \\
\cline { 2 - 5 } & Width & Length & Width & Length \\
\hline Mean difference compared with cDSA (mm) & 0.60 & 0.47 & 0.75 & 0.69 \\
\hline SD & 0.42 & 0.62 & 0.67 & 0.46 \\
\hline $95 \% \mathrm{Cl}$ & $0.37,0.65$ & $0.42,0.93$ & $0.47,1.21$ & $0.56,1.13$ \\
\hline
\end{tabular}

Abbreviations: 3D TOF MRA, three-dimensional time-of-flight sequence of magnetic resonance angiography; cDSA, cerebral digital subtraction angiography; $\mathrm{Cl}$, confidence interval; mm, millimeter; MR-IBBVI, magnetic resonance intracranial black blood vessel imaging; SD, standard deviation.

Table 4 Sensitivity and specificity of MR-IBBVI and 3D TOF MRA compared with cDSA

\begin{tabular}{|l|l|l|l|l|l|l|}
\hline \multirow{2}{*}{ Aneurysm size } & \multicolumn{2}{|c|}{$<3 \mathrm{~mm}$} & \multicolumn{2}{c|}{ 3-7 mm } & \multicolumn{2}{c|}{$>7 \mathrm{~mm}$} \\
\cline { 2 - 8 } & MR-IBBVI & 3D TOF MRA & MR-IBBVI & 3D TOF MRA & MR-IBBVI & 3D TOF MRA \\
\hline Sensitivity & 98.74 & 61.00 & 89.20 & 54.34 & 95.65 & 72.68 \\
\hline Specificity & 91.21 & 80.43 & 92.23 & 96.45 & 100.00 & 100.00 \\
\hline
\end{tabular}

Abbreviations: 3D TOF MRA, 3-dimensional time-of-flight sequence of magnetic resonance angiography; cDSA, cerebral digital subtraction angiography; MR-IBBVI, magnetic resonance intracranial black blood vessel imaging.

\section{Aneurysm Shape}

The shape of the aneurysms by MR-IBBVI (saccular-shaped, fusiform-shaped, and residual neck aneurysm) corresponded with cDSA imaging.

The mean values of sensitivity and specificity of MR-IBBVI in this study were high (98.74 and $91.21 \%$, respectively) to evaluate a small aneurysm in any location compared with the gold standard cDSA. However, 3D TOF MRA had only $61 \%$ sensitivity and $80.43 \%$ specificity (-Table 4 ).

\section{Discussion}

Neuroimaging is an important tool for evaluating patients with cerebral aneurysm. ${ }^{5}$ Each neuroimaging technique has unique strengths, weaknesses, and improvements in design and function. The three major imaging methods used for neuroimaging of cerebral aneurysms are computed tomography angiography (CTA), MRA, and the gold standard cDSA. ${ }^{3}$ cDSA can detect and reveal hemodynamic information and anatomical luminal changes, whereas MR-IBBVI can recognize and show luminal change and direct anatomical information related to the vascular wall and vascular plaque. Parenchymal information specific to the area of vascular abnormality can also be demonstrated. ${ }^{6}$ The use of 3D TOF MRA eliminates the need for administration of contrast agents, which may be contraindicated in patients with renal failure or contrast allergy; the high signal intensity of this technique can demonstrate in thrombus and hematoma. Hence, the thrombus or hematoma can be mimicked to the residual aneurysm. ${ }^{7}$

To our knowledge and based on our review of the literature, this is the first study to compare MR-IBBVI to previously accepted methods to evaluate intracranial aneurysms. Similarly, a previous literature found 3D TOF MRA could be alternative tool to cDSA for follow-up of coiled intracranial aneurysms. ${ }^{8,9}$ The precise diagnosis of 3D TOF MRA was related to the size of the aneurysm. 3D TOF MRA had limitation to detect the small aneurysm $(\leq 3 \mathrm{~mm})$ from several literature. ${ }^{10}$ In the present study, MR-IBBVI showed $98.74 \%$ sensitivity for small aneurysm localization and sizing compared with the gold standard cDSA, whereas 3D TOF MRA had 
a sensitivity of $61 \%$. 3D TOF MRA could not evaluate $15 \%$ of the aneurysms because it was unable to differentiate between residual aneurysm and thrombus due to abnormal signals of the slow flow or flow stasis as noted in a prior study ${ }^{7}$ (-Figs. 2 and 3). Moreover, in this current study, MR-IBBVI was diagnostically accurate in the ability to evaluate the size of an intracranial aneurysm. MR-IBBVI could detect a 1-mm difference in aneurysm size after a comparison with the gold standard CDSA that was relatively higher compared with 3D TOF MRA (73.3\% in width and 70\% in length). The accuracy of MR-IBBVI was found predominantly in saccular-shaped aneurysm ( $88.2 \%$ in measured width, $p=0.413$ ), size $\sim 3-7 \mathrm{~mm}$ $(91.7 \%, p=0.157)$, and aneurysm in pretreatment $(84 \%$, $p=0.650$ ) even though there was no statistical significance $(p>0.05)$. Aneurysm location was not statistically significant for an accurate measurement $(p=0.93)$. Factors that influence the interpretation error of aneurysm size are dissecting or fusiform aneurysm and a time difference of more than 1 year between MR-IBBVI and CDSA imaging. These two factors were assessed but no definite significant associated factor was found. The small population in this study precluded a multivariate analysis. Even the CTA could not be used for coiled aneurysms, but it is superior to MRA after evaluation of microsurgical clipping. ${ }^{11}$ After using the multidetector row scanners and multiparameters of imaging, CTA is able to detect small residual aneurysm and show patency or vasospasm of the adjacent parent arteries. Furthermore, CTA can demonstrate the de novo aneurysms after surgical clipping as well. However, CTA has limited ability to characterize a thrombosed aneurysm or regrowth aneurysm after coiling or clipping. Therefore, we assumed that the MR-IBBVI may also be better than CTA in this regard even though it was out of the scope of this study ( - Fig. 4). Other advantages of intracranial MR-IBBVI over the lumen-based methods previously mentioned are the detection of parenchymal lesion, residual neck aneurysm/regrowth aneurysm posttreatment, or thrombosed aneurysm. Vessel wall enhancement has recently been used to assess vasculitis or atherosclerotic plaque activity. ${ }^{12}$ The Lindenholz et $\mathrm{al}^{13}$ and Dieleman et $\mathrm{al}^{14}$ studies recommended how to use MR-IBBVI and reported the pitfalls of intracranial MR-IBBVI on intracranial vascular disease. With those two studies in mind, our study was able to show the accuracy of MR-IBBVI in measuring intracranial arterial size that may be helpful as a reference for studying other intracranial vascular diseases. As far as we know, only one study by Lee et al was an attempt on this issue on their patients with intracranial arterial stenosis. ${ }^{6}$

The limitations of our study need to be considered. It was a single-center, retrospective, nonrandomized study with too few patients to evaluate the multivariate factors that can cause inaccurate measurements of aneurysm size. In addition to the application of MR-IBBVI to detect or measure aneurysm size, MR-IBBVI could be a tool for risk stratification of multiple cerebral aneurysms to predict which aneurysm will rupture in the future. Since arterial wall inflammation plays a role in the pathophysiology of cerebral aneurysm formation, MR-IBBVI could be a surrogate marker to identify aneurysm wall enhancement on fusiform or partially thrombosed cerebral aneurysms. Therefore, aneurysmal wall enhancement is often associated with an atherosclerotic degenerative process. In contrast, if no wall or a thin wall is revealed without wall enhancement that is often invisible in MR-IBBVI, it could be safe or there is no wall vulnerability. Hence, extended studies can focus on MR-IBBVI as a modality

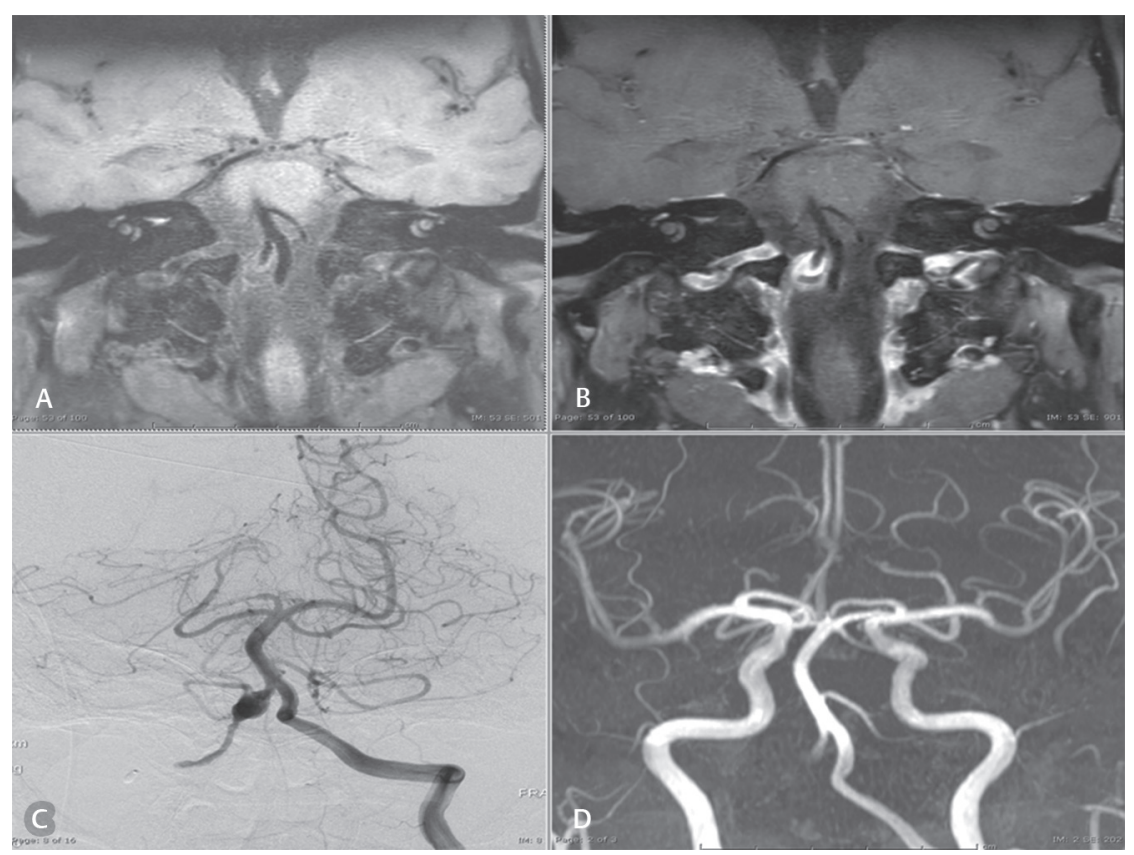

Fig. 2 Case: A 61-year-old man presented with left hemiparesis and left facial palsy. (A and B) Magnetic resonance black blood vessel imaging (T1-VISTA and T1-VISTA/Gd, respectively) show partial thrombosis with residual aneurysm. (C) Residual aneurysm using digital subtraction angiography. (D) Visualized flow or related enhancement to characterize the aneurysm using three-dimensional time-of-flight sequence of magnetic resonance angiography reconstruction. 


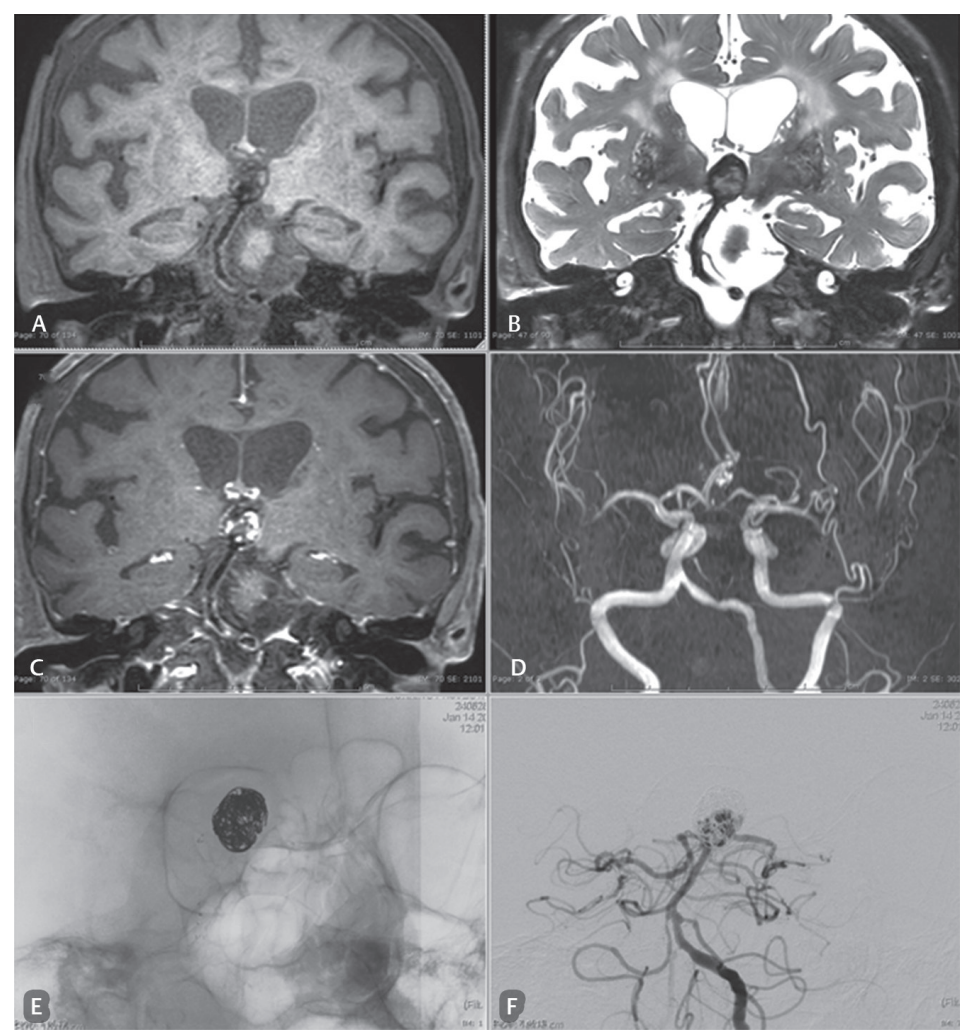

Fig. 3 Case: A 74-year-old woman presented with memory impairment. She was a follow-up patient with status post basilar tip aneurysm with stent-assisted coiling. (A-C) Magnetic resonance black blood vessel imaging (T1-VISTA, T2-VISTA/fat suppressed, T1-VISTA/Gd) that show residual/regrowth of the neck and body of a coiled basilar tip aneurysm in the area of a loosened coil mesh. The compact coil at the superior aneurysm is demonstrated. (D) The limitation of three-dimensional time-of-flight sequence of magnetic resonance angiography. Only a small part of the thrombus within the aneurysm or in between the coiled mesh is observed. There is no ability to characterize a thrombosis or residual aneurysm. (E and F) Digital subtraction angiography show a coil mesh with a little loosening at the neck and body and a regrowing aneurysm.

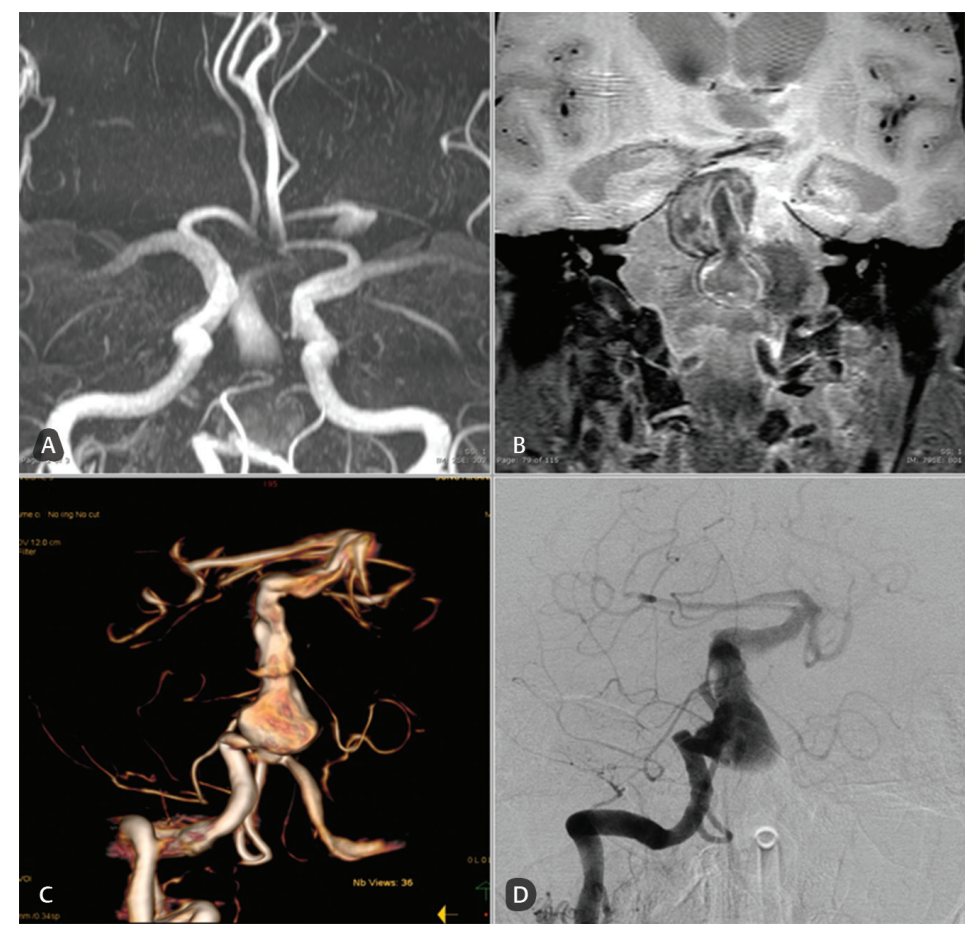

Fig. 4 Case: A 74-year-old man with a history of recurrent ischemic stroke. Diagnosis was fusiform aneurysm of the basilar artery. (A) A three-dimensional time-of-flight sequence of magnetic resonance angiography, (B) magnetic resonance black blood vessel imaging (T1-VISTA/Gd), and (C and D) digital subtraction angiography that show no significant differences in the saccular component of the basilar trunk fusiform aneurysm. 
to follow the progression of cerebral aneurysms that should provide some benefit.

\section{Conclusion}

MR-IBBVI is an accurate and highly sensitive method to detect and evaluate the size of intracranial aneurysms both before and after coiling.

\section{Highlights}

- MR-IBBVI can help in the assessment of aneurysm wall inflammation and wall thickness which may help predict the risk of aneurysm rupture.

- Diagnostic cerebral angiography is invasive procedure; otherwise MR intracranial black blood vessel imaging is noninvasive and can detect residual or regrowth aneurysm after treatment.

- MR-IBBVI is an accurate and highly sensitive method to detect and evaluate the size of an intracranial aneurysm both before and after coiling.

\section{Ethical approval}

All procedures performed in studies involving human participants were in accordance with the ethical standards of the Siriraj Institutional Review Board and with the 1964 Helsinki Declaration and its later amendments or comparable ethical standards.

\section{Conflict of Interest}

None declared.

\section{Acknowledgment}

The authors are grateful to the clinical epidemiologist in our institute for assistance with the statistical analysis.

\section{References}

1 Keedy A. An overview of intracranial aneurysms. McGill J Med 2006;9(2):141-146

2 Osborn GN, Diagnostic Imaging; Brain. 3rd edition. Philadelphia: Elsevier; 2015
3 Yoon KN, McNally S, Taussky P, et al. Imaging of cerebral aneurysms: a clinical perspective. Neurovasc Imaging 2016; 2(6):1-7

4 Mandell DM, Mossa-Basha M, Qiao Y, et al. Vessel Wall Imaging Study Group of the American Society of Neuroradiology. Intracranial vessel wall MRI: principles and expert consensus recommendations of the American society of neuroradiology. AJNR Am J Neuroradiol 2017;38(2):218-229

5 Gamal HG. Diagnostic accuracy of contrast enhancement MRI versus CTA in diagnosis of intracranial aneurysm in patients with non-traumatic subarachnoid hemorrhage. The Egyptian J Radiol Nucl Med 2015;46:125-130

6 Lee NJ, Chung MS, Jung SC, et al. Comparison of high-resolution MR imaging and digital subtraction angiography for the characterization and diagnosis of intracranial artery disease. AJNR Am J Neuroradiol 2016;37(12):2245-2250

7 Deutschmann HA, Augustin M, Simbrunner J, et al. Diagnostic accuracy of 3D time-of-flight MR angiography compared with digital subtraction angiography for follow-up of coiled intracranial aneurysms: influence of aneurysm size. AJNR Am J Neuroradiol 2007;28(4):628-634

8 de Boysson H, Boulouis G, Parienti JJ, et al. Concordance of time of flight MRA and digital subtraction angiography in adult primary central nervous system vasculitis. AJNR Am J Neuroradiol 2017;38(10):1917-1922

9 Brunereau L, Cottier JP, Sonier CB, et al. Prospective evaluation of time-of-flight MR angiography in the follow-up of intracranial saccular aneurysms treated with Guglielmi detachable coils. J Comput Assist Tomogr 1999;23(2):216-223

10 Okahara M, Kiyosue H, Yamashita M, et al. Diagnostic accuracy of magnetic resonance angiography for cerebral aneurysms in correlation with 3D-digital subtraction angiographic images: a study of 133 aneurysms. Stroke 2002;33(7):1803-1808

11 Wallace RC, Karis JP, Partovi S, Fiorella D. Noninvasive imaging of treated cerebral aneurysms, Part II: CT angiographic follow-up of surgically clipped aneurysms. AJNR Am J Neuroradiol 2007;28(7):1207-1212

12 Qiao Y, Zeiler SR, Mirbagheri S, et al. Intracranial plaque enhancement in patients with cerebrovascular events on high-spatial-resolution MR images. Radiology 2014;271(2): 534-542

13 Lindenholz A, van der Kolk AG, Zwanenburg JJ, Hendrikse $\mathrm{J}$. The use and pitfalls of intracranial vessel wall imaging: how we do it. Radiology 2018;286(1):12-28

14 Dieleman N, van der Kolk AG, Zwanenburg JJ, et al. Imaging intracranial vessel wall pathology with magnetic resonance imaging: current prospects and future directions. Circulation 2014;130(2):192-201 